\title{
INDEX OF SPECIES
}

Abraxas grossulariata, 263

Aegilops comosa, 624

Agrostis, 221-224

tenuis, 99-108, 206-217, 220, 226, 227, 237, 522

Alauda arvensis, 177

Allium, 45, 591-605

angulosum, 592, 594

ascolonicium, 591

azureum, 592, 594

сера, 591-594, 599-602

cernum, 592, 594, 597

darwasicum, 592, 594

dicipience, 592, 594

fistulosum, 591, 592, 594, 599-602

fragrans, 592

fuscum, 592, 594, 597

galanthum, 592, 594

globosum, 592, 594

hirsutum, 592, 594

jesolianum, 592, 594

karataviense, 592, 594, 596

margaritaceum, 592, 594

moly, 592, 597

neapolitanum, 592, 594, 597

odoratissimum, 592, 594

pulchellum, 592

roseum, 592, 594

schoenoprasum, 592, 594

senescens, 592, 594

sibiricum, 592, 594

stellatum, 592, 594

subhirsutum, 592, 594

triquetrum, 592, 594

ursinum, 592, 597

yunnanenes, 592

zebdanense, 592, 594, 596

Anadara trapezia, 485

Anisantha sterilis, 172

Anthoxanthum, 101, 221, 222, 223, 224, 235, 236

odoratum, 206-217, 220, 237

Antirrhinum magus, 67-72

Arabidopsis thaliana, 127-141, 321-337, 539589

Arachis hypogea, 335

Aristelliger praesignis, 433

Ascobolus immersus, 474

Aspergillus, 443

nidulans, 164, 165, 443-452, 467, 626, 627

unguis, 451

Bacillus subtilis, 476

Biston betularia, 263

Blaberus, 73, 78

Brassica oleracea, 119-125
Campanula, 73

perisicifolia, 74

Canna, 73, 79

Carduus vulgare, 172

Centaurea nigra, 172

Cepaea, 81, 88, 96

hortensis, 169-188

nemoralis, 169-188

Chironomus, 602, 603

thummi thumii, 602

Chlamydomonas reinhardi, 143, 144

Chorthippus parallelus, 630

Clarkia, 219

xantiana, 213

Coenonympha tullia, 611-614

Collybia velutipes, 571

Coprinus lagopus, 109

Crepis, 604

Dactylis, 279-300, 369-386

glomerata, 172

Dictyostelium discoideum, 474

Drosophila, 1, 14, 15, 20, 45, 135, 136, 204, 205, 263-278, 335, 364, 387, 392, 418, $499,621,630$

melanogaster, 127, 199, 202, 203, 263, 321$337,400,433-442,553,563-572$

persimilis, 199

pseudobscura, 199, 202

robusta, 199

virilis, 199, 202

willistoni, 199-204

Epilobium hirsutum, 366, 367

Escherichia coli, 166, 469, 470, 471, 476

Festuca, 101

ovina, 207, 365, 522

rubra, 172

Gadus morhua, 629

Galeopis, 587

Galium, 101

Geranium robertianum, 213

Geum rivale, 213

Geum urbane, 213

Gilia, 205

capitata, 213

Gramineae, 624

Hemizonia citrina, 213

lutescens, 213

luzulaefolia, 213

rudis, 213

Homo sapiens, 317 
Ixeris denticulata, 213

Lactuca canadensis, 213 graminifolia, 213

Lamium amplexicaule, 213

Lathyrus, 603, 604

Layia platyglossa, 213

Leontodon autumnale, 213

Lilium longiflorum, 143

Limicolaria, 81 aurora, 81

flammulata, 81 martensiana, 97

Linum, 25, 301-310

Lolium, 37, 101, 297, 299, 382, 603 perenne, 357-368, 624

Lotus corniculatus, 172, 453-455

Luzula, 45

Lycopersicum, 247-256, 382 esculentum, 135, 247-256 pimpinellifolium, 135

Madia elegans, 213

Maniola jurtina, 205

Medicago sativa, 172

Melandrium, 382, 587

Mimulus guttatus, 213, 244

Natrix natrix, 433

Neurospora, 387, 400 crassa, 162-163, 168, 443, 450, 627

Nicotiana, 220, 400, 602 rustica, 135, 339, 527, 571

Oenanthe oenanthe, 177

Oenothera, 73

Papaver, 587

dubium, 571

Papilio, 263

dardanus, 205

Paramecium, 165, 628 aurelia, 628

Paratettix texanus, 96

Partula suturalis, 189-198 taeniata, 189-198

Periplaneta, 73, 78

Phymateus cinctus, 607-611

Physarum polycephalum, 474

Picris echoides, 172

Pinus, 382 attenuata, 213 radiata, 213

Pisum, 382

Plantago lanceolata, 172

Populus, 243

Pteridium, 101

Puccinia striiformis, 624

Puschkinia libanotica, 625

Ranunculus acer, 213

Rhizobium trifolii, 537, 538, 548, 551
Rhoeo, 73

Rumex acetosa, 213

Saccharomyces cerevisiae, 467-468, 468, 475

Salmonella, 470

Salvia apiana, 213 mellifera, 213

Schizophyllum commune, 109-117, 387-402

Schizosaccharomyces pombe, 163

Secale, 297, 382 cereale, 73

Silene cucubalis, 213 inflata, 207 maritima, 213

Solanum, 205 tuberosum, 625

Solidago virgaurea, 213

Sordaria fimicola, 474

Sorghum aethipicum, 1-23 almum, 1-23

arundinaceum, 1-23

bicolor, 1-23

brevicarinatum, 1-23

caffrorum, 1-23

cordatum, 1-23

durra, 1-23

guineense, 1-23

halepense, 1-23

macrochaeta, 1-23

purpureo-sericeum, 1-23

roxburghii, 1-23

sudanense, 1-23

versicolor, 1-23

verticilli florum, 1-23

virgatum, 1-23

Streptanthus, 205

Streptocarpus, 366, 367

Streptomyces coelicolor, 628

Succisa pratensis, 213

Tetrahymena, 628

Tradescantia paludosa, 143

Tribolium castaneum, 477

Trifolium repens, 453-455

Triphaena comes, 263

Tripsacum, 18, 19

Triticum, 382

aestivum, 623

Triturus, 45

Turdus ericetorum, 177

Ustilago maydis, 471

Vicia, 220

faba, 591

sativa, 591

Viola, 141 tricolor, 213

Vitis, 140

Zea, 18, 19 mays, 39-47

perennis, 20 